\title{
OSCILLATION OF A CLASS OF THIRD ORDER GENERALIZED FUNCTIONAL DIFFERENCE EQUATION P.Venkata Mohan Reddy ${ }^{1}$, Adem Kılıçman ${ }^{2}$ M.Maria Susai Manuel ${ }^{3}$. \\ ${ }_{1,3}$ Department of Science and Humanities, R. M. D. Engineering College, Kavaraipettai - 601 206, Tamil Nadu, S. India \\ ${ }^{2}$ Department of Mathematics and Institute for Mathematical Research, University Putra Malaysia, 43400 UPM, Serdang, Selangor, Malaysia.
}

\section{Abstract}

The authors intend to establish new oscillation criteria for a class of generalized third order functional difference equation of the form

$$
\Delta_{\ell}\left(a_{2}(n)\left[\Delta_{\ell}\left(a_{1}(n)\left[\Delta_{\ell} z(n)\right]^{\beta_{1}}\right)\right]^{\beta_{2}}\right)+q(n) f(x(g(n)))=0, n \geq n_{0},
$$

where $z(n)=x(n)+p(n) x(\tau(n))$. We also present sufficient conditions for the solutions to converges to zero. Suitable examples are presented to validate our main results.

Keywords: Generalized difference operator, Oscillation, Convergence.

AMS Subject Classification [2000]: 39A10, 39A11.

\section{INTRODUCTION}

Difference equations usually occur due to certain phenomena over time, and it play an important role in describing discrete dynamical systems [1]. Difference equation and their associated operators not only play a role in their own right as direct mathematical models of physical phenomena but also provide the field of numerical analysis with powerful tools. Difference equations also occur in combined form with differential equations, commonly called differential-difference equations yielding rich models, particularly in control theory. Difference equations are widely used in the theory of probability, biology, engineering, social and behavioral sciences.

Difference equations are evolved normally concerning the operator $\Delta$ and its higher orders defined as

$$
\Delta x(n)=x(n+1)-x(n), \quad n \in \mathbb{Z}=\{0,1,2,3, \cdots\}
$$


A few authors ([1], [10], [13]) also defined the operator $\Delta$ as

$$
\Delta x(n)=x(n+\ell)-x(n),
$$

$\ell$ is a positive integer. None has consider the definition of $\Delta$ given in (3) for further study on the theory of difference equations because of the difficulty involved when $\ell$ is different from unity. Recently, Adem Kilicman, Thandapani, Maria Susai Manuel and Britto Antony Xavier considered the definition of $\Delta$ as given in (3) and derived exiting results on number theory ([5]-[7]) by denoting the new operator as $\Delta_{\ell}$. Also new oscillation criteria and new asymptotic properties are obtained by considering difference equations involving $\Delta_{\ell}$.

Oscillation is one of the main topics of interest in the study of difference equations. Active research is on in the last few decades in analyzing the oscillatory behavior of the solution of difference equations involving $\Delta$ but the study of the same property of difference equations involving $\Delta_{\ell}$ is rare. The present research is focused on establishing new oscillation criteria of the class of third order generalized difference equation given in (1). We also present sufficient conditions for the solution to converge to zero. For the theory related to the relevant topic, one can refer ([2], [4, [8], [9], [1], [12] ).

In this paper, we deal with the oscillation and the asymptotic behavior of solutions of the third-order generalized functional difference equation of the form

$$
\Delta_{\ell}\left(a_{2}(n)\left[\Delta_{\ell}\left(a_{1}(n)\left[\Delta_{\ell} z(n)\right]^{\beta_{1}}\right)\right]^{\beta_{2}}\right)+q(n) f(x(g(n)))=0, n \geq n_{0},
$$

where $z(n)=x(n)+p(n) x(\tau(n))$.

We make the following assumptions throughout this paper.

(a) $\mathbb{N}=\{0,1,2,3, \ldots\}, \mathbb{N}(a)=\{a, a+1, a+2, \ldots\}$,

(b) $\mathbb{N}_{\ell}(a)=\{a, a+\ell, a+2 \ell, \ldots\}$.

(c) $\lceil x\rceil=$ upper integer part of $x$ and $[x]=$ integer part of $x$.

(d) $j=n-n_{i}-\left[\frac{n-n_{i}}{\ell}\right] \ell, n_{i} \in[0, \infty)$.

(e) $\left\{a_{i}(n)\right\}$ is a positive increasing sequence of real numbers for all $n \geq n_{0}$ and satisfies the condition $\sum_{s=n_{0}}^{\infty} \frac{1}{a_{i}^{1 / \beta_{i}}(s)}=\infty, i=1,2$.

(f) $\{p(n)\}$ and $\{q(n)\}$ with $0 \leq p(n) \leq p<1, q(n)>0$ are sequences of real numbers for all $n \geq n_{0}$. 
(g) $\{\tau(n)\}$ and $\{g(n)\}$ are sequences of integers with $\tau(n) \leq n, g(n) \leq n, \Delta_{\ell} g(n)>0$, $\lim _{n \rightarrow \infty} \tau(n)=\infty$ and $\lim _{n \rightarrow \infty} g(n)=\infty$.

(h) $\beta_{1}$ and $\beta_{2}$ are a quotient of odd positive integers with $\beta=\beta_{1} \beta_{2}$.

(i) $f$ is a continuous real valued function such that $\frac{f(x)}{x^{\beta}} \geq k>0$ for $x \neq 0$ and $k$ is a constant.

\section{Preliminaries}

In this section, we present some preliminaries which will be useful for future discussion.

Definition 2.1. [5] Let $x(n), n \in[0, \infty)$ be a real or complex valued function and $\ell \in(0, \infty)$. We define, the generalized difference operators $\Delta_{\ell}$ as

$$
\Delta_{\ell} x(n)=x(n+\ell)-x(n) .
$$

Definition 2.2. [5] Let $x(n), n \in[0, \infty)$ be a real or complex valued function and $\ell \in(0, \infty)$. Then, the inverse of $\Delta_{\ell}$ denoted by $\Delta_{\ell}^{-1}$ is defined as follows.

$$
\begin{gathered}
\text { If } \Delta_{\ell} y(n)=x(n), \text { then } y(n)=\Delta_{\ell}^{-1} x(n)+c_{j}, \\
(\text { or }) \\
y(n)=y\left(n_{0}+j\right)+\sum_{r=0}^{\left[\frac{n-n_{0}-j-\ell}{\ell}\right]} x\left(n_{0}+j+r \ell\right),
\end{gathered}
$$

where $c_{j}$ is a constant for all $n \in \mathbb{N}_{\ell}(j), j=n-\left[\frac{n}{\ell}\right] \ell$.

Definition 2.3. [5] For $\lambda \in \mathbb{N}(1)$, the generalized polynomial factorial is defined by

$$
n_{\ell}^{(\lambda)}=n(n-\ell)(n-2 \ell) \cdots(n-(\lambda-1) \ell)
$$

Lemma 2.4. [5] Let $\ell \in[0, \infty)$. then $\Delta_{\ell}\left(n_{\ell}^{(\lambda)}\right)=(\lambda \ell) n_{\ell}^{(\lambda-1)}$

Lemma 2.5. Let $x(n)$ and $y(n)$ be any two real valued functions. Then

$$
\Delta_{\ell}\{x(n) y(n)\}=x(n+\ell) \Delta_{\ell} y(n)+y(n) \Delta_{\ell} x(n)=y(n+\ell) \Delta_{\ell} x(n)+x(n) \Delta_{\ell} y(n) .
$$


Definition 2.6. The solution $x(n)$ of $(1)$ is called oscillatory if for any $n_{1} \in[a, \infty)$ there exists an $n_{2} \in \mathbb{N}_{\ell}\left(n_{1}\right)$ such that $x\left(n_{2}\right) x\left(n_{2}+\ell\right) \leq 0$. The difference equation itself is called oscillatory if all its solutions are oscillatory. If the solution $x(n)$ is not oscillatory, then it is said to be nonoscillatory (i.e. $x(n) x(n+\ell)>0$ for all $\left.n \in\left[n_{1}, \infty\right)\right)$.

Lemma 2.7. [3] If $x$ and $y$ are positive and unequal, then

$$
\begin{array}{ll}
r y^{r-1}(x-y)<x^{r}-y^{r}<r x^{r-1}(x-y), & r<0 \text { or } r>1 \\
r x^{r-1}(x-y)<x^{r}-y^{r}<r y^{r-1}(x-y), & 0<r<1
\end{array}
$$

There is obviously equality when $r=0, r=1$ or $x=y$.

\section{Main Results}

We establish in this section, some new oscillation criteria for solutions of the equation (1). For the sake of convenience, we introduce the following notations.

$$
\begin{aligned}
& E_{0}(n)=z(n), E_{i}(n)=a_{i}(n)\left(\Delta_{\ell} E_{i-1}(n)\right)^{\beta_{i}}, \quad i=1,2 \\
& R_{N}(n)=\frac{1}{a_{1}^{1 / \beta_{1}}(n)}\left(\sum_{r=0}^{\frac{n-N-\ell-j}{\ell}} \frac{1}{\left[a_{2}(N+j+r \ell)\right]^{1 / \beta_{2}}}\right)^{1 / \beta_{1}} \text { and } \\
& \overline{R_{N}}(n)=\sum_{r=0}^{\frac{n-N-\ell-j}{\ell}} R_{N}(N+j+r \ell) .
\end{aligned}
$$

First, we state and prove some useful lemmas.

Lemma 3.1. Let $\{x(n)\}$ be a positive solution of equation (1). Then $z(n)$ has only one of the following two properties eventually

(i) $z(n)>0, \Delta_{\ell} z(n)>0$ and $\Delta_{\ell} E_{1}(n)>0$,

(ii) $z(n)>0, \Delta_{\ell} z(n)<0$ and $\Delta_{\ell} E_{1}(n)>0$.

Proof. Let $\{x(n)\}$ be a positive solution of equation (1). From $(g)$, there exists an $n_{1} \geq n_{0}$ such that $x(n)>0, x(\tau(n))>0$ and $x(g(n))>0$ for $n \geq n_{1}$. Then $z(n)>0$ and equation (1) implies that

$$
\Delta_{\ell} E_{2}(n)=-q(n) f(x(g(n))) \leq 0 .
$$


Hence, $E_{2}(n)$ is a non-increasing function and of one sign. We claim that $E_{2}(n)>0$ for $n \geq n_{1}$. Suppose that $E_{2}<0$ for $n \geq n_{2} \geq n_{1}$, then there exists an $n_{3} \geq n_{2}$ and a constant $K_{1}>0$ such that

$$
\Delta_{\ell} E_{1}(n)<-K_{1}\left[a_{2}(n)\right]^{-1 / \beta_{2}}<0, \text { for } n \geq n_{3} .
$$

Hence, by equation (6)

$$
E_{1}(n) \leq E_{1}\left(n_{3}+j\right)-K_{1} \sum_{r=0}^{\frac{n-n_{3}-\ell-j}{\ell}} \frac{1}{\left[a_{2}\left(n_{3}+j+r \ell\right)\right]^{1 / \beta_{2}}} .
$$

Letting $n \rightarrow \infty$, from $(e)$ we have $\lim _{n \rightarrow \infty} E_{1}(n)=-\infty$. Then there exists a $n_{4} \geq n_{3}$ and a constant $K_{2}>0$ such that

$$
\Delta_{\ell} z(n)<-K_{2}\left[a_{1}(n)\right]^{-1 / \beta_{1}}, \text { for } n \geq n_{4}
$$

Hence, by equation (6) and using $(e)$, we get $\lim _{n \rightarrow \infty} z(n)=-\infty$, which contradicts $z(n)>0$. Now we have $E_{2}(n)>0$ for $n \geq n_{1}$. Therefore, $E_{1}(n)$ is an increasing function. Thus property (i) or property $(i i)$ holds for $z(n)$ eventually.

Lemma 3.2. Let $\{x(n)\}$ be a positive solution of equation (1), and $z(n)$ has the property (ii). Assume that

$$
\sum_{t=0}^{\infty} \frac{1}{a_{1}^{1 / \beta_{1}}\left(n_{3}+j+t \ell\right)}\left[\sum_{s=0}^{\frac{t-n_{2}-\ell-j}{\ell}} \frac{1}{a_{2}^{1 / \beta_{2}}\left(n_{2}+j+s \ell\right)}\left[\sum_{r=0}^{\frac{s-n_{1}-\ell-j}{\ell}} q\left(n_{1}+j+r \ell\right)\right]^{\frac{1}{\beta_{2}}}\right]^{\frac{1}{\beta_{1}}}=\infty
$$

Then, the solution $\{x(n)\}$ of equation (1) converges to zero as $n \rightarrow \infty$.

Proof. Let $\{x(n)\}$ be a positive solution of equation (1). Since $z(n)$ satisfies the property $(i i)$, we get

$$
\lim _{n \rightarrow \infty} z(n)=\gamma \geq 0
$$

Now, we shall prove that $\gamma=0$. Let $\gamma>0$, then we have $\gamma<z(n)<\gamma+\epsilon$ for all $\epsilon>0$ and $n$ sufficiently large. Choosing $\epsilon<\frac{1-p}{p} \gamma$, we obtain

$$
x(n)=z(n)-p(n) x(\tau(n))>\gamma-p(n) z(\tau(n))>L(\gamma+\epsilon)>L z(n),
$$


where $L=\frac{\gamma-p(\gamma+\epsilon)}{\gamma+\epsilon}>0$. Hence, from equation 11 and $(i)$, we have

$$
\Delta_{\ell} E_{2}(n) \leq-k q(n) x^{\beta}(g(n))<-k L^{\beta} q(n) z^{\beta}(g(n))<-k L^{\beta} \gamma^{\beta} q(n)
$$

Therefore, by equation (6), summing this inequality form $n_{1}$ to $\infty$, we get

$$
\Delta_{\ell} E_{1}(n)>-k^{\frac{1}{\beta_{2}}} L^{\frac{\beta}{\beta_{2}}} \gamma^{\frac{\beta}{\beta_{2}}} \frac{1}{a_{2}^{1 / \beta_{2}}(n)}\left(\sum_{r=0}^{\frac{n-n_{1}-\ell-j}{\ell}} q\left(n_{1}+j+r \ell\right)\right)^{1 / \beta_{2}} .
$$

Summing again form $n_{2}$ to $\infty$, we obtain

$$
\Delta_{\ell} z(n)<\frac{-C}{a_{1}^{1 / \beta_{1}}(n)}\left(\sum_{s=0}^{\frac{n-n_{2}-\ell-j}{\ell}} \frac{1}{a_{2}^{1 / \beta_{2}}\left(n_{2}+j+s \ell\right)}\left(\sum_{r=0}^{\frac{s-n_{1}-\ell-j}{\ell}} q\left(n_{1}+j+r \ell\right)\right)^{1 / \beta_{2}}\right)^{1 / \beta_{1}}
$$

where $C=k^{\frac{1}{\beta}} L \gamma$. Summing the last inequality from $n_{3}$ to $\infty$, we have

$$
z(n)>-C \sum_{t=0}^{\infty} \frac{1}{a_{1}^{1 / \beta_{1}}\left(n_{3}+j+t \ell\right)}\left[\sum_{s=0}^{\frac{t-n_{2}-\ell-j}{\ell}} \frac{1}{a_{2}^{1 / \beta_{2}}\left(n_{2}+j+s \ell\right)}\left[\sum_{r=0}^{\frac{s-n_{1}-\ell-j}{\ell}} q\left(n_{1}+j+r \ell\right)\right]^{\frac{1}{\beta_{2}}}\right]^{\frac{1}{\beta_{1}}} .
$$

This contradicts condition 10$)$. Hence, $\lim _{n \rightarrow \infty} z(n)=0$, which implies that $\lim _{n \rightarrow \infty} x(n)=0$.

Lemma 3.3. Let $\{x(n)\}$ be a positive solution of equation (1), and $z(n)$ has the property $(i)$. Then we have

$$
\begin{gathered}
\Delta_{\ell} E_{2}(n) \leq-k q(n)(1-p(g(n)))^{\beta} z^{\beta}(g(n)), \\
\Delta_{\ell} z(g(n)) \geq E_{2}^{1 / \beta}(n) R_{n_{0}}(g(n))
\end{gathered}
$$

and

$$
\bar{R}_{n_{0}}^{\beta}(g(n)) \frac{E_{2}(n)}{z^{\beta}(g(n))} \leq 1
$$

Proof. Let $\{x(n)\}$ be a positive solution of equation (1). From $(g)$, there exists an $n_{1} \geq n_{0}$ such that $x(n)>0, x(\tau(n))>0$ and $x(g(n))>0$ for $n \geq n_{1}$. Since $z(n)$ satisfies the property (i), we have

$$
x(n)=z(n)-p(n) x(\tau(n)) \geq(1-p(n)) z(n) .
$$


Thus, by equation (1) and $(i)$, we have

$$
\Delta_{\ell} E_{2}(n) \leq-k q(n) x^{\beta}(g(n)) \leq-k q(n)(1-p(g(n)))^{\beta} z^{\beta}(g(n))<0
$$

Again, from property (i), there exists an $N \geq n_{0}$ such that

$$
E_{1}(n)=E_{1}(N+j)+\sum_{r=0}^{\frac{n-N-\ell-j}{\ell}} \frac{E_{2}^{1 / \beta_{2}}(N+j+r \ell)}{\left[a_{2}(N+j+r \ell)\right]^{1 / \beta_{2}}} .
$$

Since $\Delta_{\ell} E_{2}(n)<0$, we obtain

$$
E_{1}(n) \leq E_{2}^{1 / \beta_{2}}(n) \sum_{r=0}^{\frac{n-N-\ell-j}{\ell}} \frac{1}{\left[a_{2}(N+j+r \ell)\right]^{1 / \beta_{2}}} .
$$

This implies that

$$
\Delta_{\ell} z(n) \geq E_{2}^{1 / \beta}(n) R_{N}(n)
$$

Since $g(n) \leq n$, we have

$$
\Delta_{\ell} z(g(n)) \geq E_{2}^{1 / \beta}(n) R_{N}(g(n)) .
$$

By summing the inequality 14 from $N$ to $n$ and using $\Delta_{\ell} E_{2}(n)<0$, we get

$$
z(n)=z(N+j)+E_{2}^{1 / \beta}(n) \sum_{r=0}^{\frac{n-N-\ell-j}{\ell}} R_{N}(N+j+r \ell) .
$$

This implies that

$$
z(n) \leq E_{2}^{1 / \beta}(n) \overline{R_{N}}(n)
$$

Thus, we get

$$
z(g(n)) \geq E_{2}^{1 / \beta}(n) \bar{R}_{N}(g(n))
$$

and so

$$
\bar{R}_{N}^{\beta}(g(n)) \frac{E_{2}(n)}{z^{\beta}(g(n))} \leq 1
$$

This completes the proof.

Remark 3.4. For simplicity, we introduce the following notations.

$$
P=\liminf _{n \rightarrow \infty} \bar{R}_{n_{0}}^{\beta}(g(n+j+\ell)) \sum_{s=n}^{\infty} \phi(n+j+s \ell)
$$


and

$$
Q=\limsup _{n \rightarrow \infty} \frac{1}{\bar{R}_{n_{0}}(g(n+j+\ell))} \sum_{s=0}^{\left[\frac{n-n_{0}-j-\ell}{\ell}\right]} \bar{R}_{n_{0}}^{\beta+1}\left(g\left(n_{0}+j+s \ell+\ell\right)\right) \phi\left(n_{0}+j+s \ell\right),
$$

where $\phi(n)=k q(n)(1-p(g(n)))^{\beta}$. Moreover for $z(n)$ satisfying property $(i)$, we define

$$
\omega(n)=\frac{E_{2}(n)}{z^{\beta}(g(n))}
$$

and

$$
\begin{aligned}
l & =\liminf _{n \rightarrow \infty} \bar{R}_{n_{0}}^{\beta}(g(n+j+\ell)) \omega(n+j+\ell), \\
U & =\limsup _{n \rightarrow \infty} \bar{R}_{n_{0}}^{\beta}(g(n+\ell)) \omega(n) .
\end{aligned}
$$

Lemma 3.5. Let $\{x(n)\}$ be a positive solution of equation (1).

(1) Let $P<\infty, Q<\infty$ and $z(n)$ satisfies property $(i)$. If

$$
\lim _{n \rightarrow \infty} \bar{R}_{n_{0}}(n)=\infty
$$

then

$$
P \leq l-l^{\frac{1+\beta}{\beta}} \text { and } P+Q \leq 1
$$

(2) If $P=\infty$ or $Q=\infty$, then $z(n)$ does not have property $(i)$.

Proof. Part(1); Let $\{x(n)\}$ be a positive solution of equation (1) and $z(n)$ satisfies property (i). By Lemma 3.3, we have that (11), 12) and (13) hold. From the definition of $\omega(n)$, we see that $\omega(n)$ is positive and satisfies

$$
\Delta_{\ell} \omega(n)=\frac{\Delta_{\ell} E_{2}(n)}{z^{\beta}(g(n))}-\frac{E_{2}(n+\ell) \Delta_{\ell} z^{\beta}(g(n))}{z^{\beta}(g(n)) z^{\beta}(g(n+\ell))} .
$$

Now, by using equation (8), we find that

$$
\Delta_{\ell} z^{\beta}(g(n))=z^{\beta}(g(n+\ell))-z^{\beta}(g(n))<\beta z^{\beta-1}(g(n+\ell)) \Delta_{\ell} z(g(n)) .
$$

The equation (19) can be rewritten as

$$
\Delta_{\ell} \omega(n)=\frac{\Delta_{\ell} E_{2}(n)}{z^{\beta}(g(n))}-\frac{\beta E_{2}(n+\ell) \Delta_{\ell} z(g(n))}{z^{\beta}(g(n)) z(g(n+\ell))} .
$$


Thus, from (11) and (12), there exists an $N \geq n_{0}$ such that

$$
\Delta_{\ell} \omega(n) \leq-k q(n)(1-p(g(n)))^{\beta}-\frac{\beta E_{2}^{\frac{1+\beta}{\beta}}(n+\ell) R_{N}(g(n+\ell))}{z^{1+\beta}(g(n+\ell))} .
$$

for $n \geq N$. This implies that

$$
\Delta_{\ell} \omega(n) \leq-\phi(n)-\beta R_{N}(g(n+\ell)) \omega^{\frac{1+\beta}{\beta}}(n+\ell) .
$$

From (13), we get

$$
\bar{R}_{N}^{\beta}(g(n)) \omega(n) \leq 1,
$$

which with 177 gives

$$
\lim _{n \rightarrow \infty} \omega(n)=0 .
$$

On the other hand, from the definition of $\omega(n), l$ and $U$, we see that

$$
0 \leq l \leq U \leq 1
$$

Now, we prove that the first ineqality in $(18)$ holds. Let $\epsilon>0$, then from the definitions of $P$ and $l$, we can choose $n_{2} \geq N$ sufficiently large that

$$
\begin{aligned}
& \bar{R}_{N}^{\beta}(g(n+j+\ell)) \sum_{s=0}^{\infty} \phi(n+j+s \ell) \geq P-\epsilon \\
& \text { and } \quad \bar{R}_{N}^{\beta}(g(n+j+\ell)) \omega(n+j+\ell) \geq l-\epsilon \quad \text { for } \quad n \geq n_{2} .
\end{aligned}
$$

By summing 21) from $n$ to $\infty$ and using 22, we have

$$
\omega(n+j) \geq \sum_{s=0}^{\infty} \phi(n+j+s \ell)+\beta \sum_{s=0}^{\infty} R_{N}(g(n+j+s \ell+\ell)) \omega^{\frac{1+\beta}{\beta}}(n+j+s \ell+\ell) .
$$

Multiplying the above inequality by $\bar{R}_{N}^{\beta}(g(n+j+\ell))$, we obtain

$$
\begin{aligned}
& \bar{R}_{N}^{\beta}(g(n+j+\ell)) \omega(n+j) \geq \omega(n+j) \geq \bar{R}_{N}^{\beta}(g(n+j+\ell)) \sum_{s=0}^{\infty} \phi(n+j+s \ell) \\
& +\beta \bar{R}_{N}^{\beta}(g(n+j+\ell)) \sum_{s=0}^{\infty} \frac{R_{N}(g(n+j+s \ell+\ell))}{\bar{R}_{N}^{1+\beta}(g(n+j+s \ell+\ell))}\left(\bar{R}_{N}^{\beta}(g(n+j+s \ell+\ell)) \omega(n+j+s \ell+\ell)\right)^{\frac{1+\beta}{\beta}} . \\
& \geq(P-\epsilon)+(l-\epsilon)^{\frac{1+\beta}{\beta}} \beta \bar{R}_{N}^{\beta}(g(n+j+\ell)) \sum_{s=0}^{\infty} \frac{R_{N}(g(n+j+s \ell+\ell))}{\bar{R}_{N}^{1+\beta}(g(n+j+s \ell+\ell))} . \\
& \geq(P-\epsilon)+(l-\epsilon)^{\frac{1+\beta}{\beta}} .
\end{aligned}
$$


Taking limit inferior on both sides as $n \rightarrow \infty$, we get

$$
l \geq(P-\epsilon)+(l-\epsilon)^{\frac{1+\beta}{\beta}}
$$

Since $\epsilon>0$ is arbitrary, we obtain the desired result

$$
P \leq l-l^{\frac{1+\beta}{\beta}}
$$

Next, we prove the second inequality in part (1). Multiplying 21 by $\bar{R}_{N}^{\beta+1}(g(n+\ell))$ and summing it from $n_{2}$ to $n-\ell$, we obtain

$$
\begin{aligned}
& \quad \sum_{s=0}^{\frac{n-n_{2}-j-\ell}{\ell}} \bar{R}_{N}^{\beta+1}\left(g\left(n_{2}+j+s \ell+\ell\right)\right) \Delta_{\ell} \omega\left(n_{2}+j+s \ell\right) \\
& \leq-\sum_{s=0}^{\frac{n-n_{2}-j-\ell}{\ell}} \bar{R}_{N}^{\beta+1}\left(g\left(n_{2}+j+s \ell+\ell\right)\right) \phi\left(n_{2}+j+s \ell\right) \\
& \quad-\beta \sum_{s=0}^{\frac{n-n_{2}-j-\ell}{\ell}} R_{N}\left(g\left(n_{2}+j+s \ell+\ell\right)\right)\left(\bar{R}_{N}^{\beta}\left(g\left(n_{2}+j+s \ell+\ell\right)\right) \omega\left(n_{2}+j+s \ell+\ell\right)\right)^{\frac{1+\beta}{\beta}} .
\end{aligned}
$$

By Summation by parts, we obtain

$$
\begin{aligned}
\bar{R}_{N}^{\beta+1}(g(n+\ell)) \omega(n) \leq \bar{R}_{N}^{\beta+1}\left(g\left(n_{2}+j+\ell\right)\right) \omega\left(n_{2}+j\right) \\
\quad+\sum_{s=0}^{\frac{n-n_{2}-j-\ell}{\ell}} \omega\left(n_{2}+j+s \ell+\ell\right) \Delta_{\ell} \bar{R}_{N}^{\beta+1}\left(g\left(n_{2}+j+s \ell+\ell\right)\right) \\
\quad-\quad \sum_{s=0}^{\frac{n-n_{2}-j-\ell}{\ell}} \bar{R}_{N}^{\beta+1}\left(g\left(n_{2}+j+s \ell+\ell\right)\right) \phi\left(n_{2}+j+s \ell\right) \\
\quad-\beta \sum_{s=0}^{\frac{n-n_{2}-j-\ell}{\ell}} R_{N}\left(g\left(n_{2}+j+s \ell+\ell\right)\right)\left(\bar{R}_{N}^{\beta}\left(g\left(n_{2}+j+s \ell+\ell\right)\right) \omega\left(n_{2}+j+s \ell+\ell\right)\right)^{\frac{1+\beta}{\beta}}
\end{aligned}
$$


Hence,

$$
\begin{aligned}
\bar{R}_{N}^{\beta+1}(g(n+\ell)) \omega(n) \leq & \bar{R}_{N}^{\beta+1}\left(g\left(n_{2}+j+\ell\right)\right) \omega\left(n_{2}+j\right) \\
& -\sum_{s=0}^{\frac{n-n_{2}-j-\ell}{\ell}} \bar{R}_{N}^{\beta+1}\left(g\left(n_{2}+j+s \ell+\ell\right)\right) \phi\left(n_{2}+j+s \ell\right) \\
& +\sum_{s=0}^{\frac{n-n_{2}-j-\ell}{\ell}} R_{N}\left(g\left(n_{2}+j+s \ell+\ell\right)\right)\left((\beta+1) M-\beta M^{\frac{1+\beta}{\beta}}\right),
\end{aligned}
$$

where $M=\bar{R}_{N}^{\beta}\left(g\left(n_{2}+j+s \ell+\ell\right)\right) \omega\left(n_{2}+j+s \ell+\ell\right)$.

Using the inequalaity

$$
A u-B u^{\frac{1+\beta}{\beta}} \leq \frac{\beta^{\beta}}{(1+\beta)^{1+\beta}} \frac{A^{1+\beta}}{B^{\beta}}
$$

with $u=M, A=(1+\beta)$ and $B=\beta$, we obtain

$$
\begin{aligned}
\bar{R}_{N}^{\beta+1}(g(n+\ell)) \omega(n) \leq & \bar{R}_{N}^{\beta+1}\left(g\left(n_{2}+j+\ell\right)\right) \omega\left(n_{2}+j\right) \\
& \quad-\sum_{s=0}^{\frac{n-n_{2}-j-\ell}{\ell}} \bar{R}_{N}^{\beta+1}\left(g\left(n_{2}+j+s \ell+\ell\right)\right) \phi\left(n_{2}+j+s \ell\right)+\bar{R}_{N}(g(n+j+\ell)) .
\end{aligned}
$$

It follows that

$$
\begin{aligned}
\bar{R}_{N}^{\beta}(g(n+\ell)) \omega(n) & \leq \frac{\bar{R}_{N}^{\beta+1}\left(g\left(n_{2}+j+\ell\right)\right) \omega\left(n_{2}+j\right)}{\bar{R}_{N}(g(n+j+\ell))} \\
& -\frac{1}{\bar{R}_{N}(g(n+j+\ell))} \sum_{s=0}^{\frac{n-n_{2}-j-\ell}{\ell}} \bar{R}_{N}^{\beta+1}\left(g\left(n_{2}+j+s \ell+\ell\right)\right) \phi\left(n_{2}+j+s \ell\right)+1 .
\end{aligned}
$$

Taking limit superior on both sides as $n \rightarrow \infty$ and using (17) we get

$$
U \leq 1-Q
$$

Thus, form (23), we have

$$
P \leq l-l^{\frac{1+\beta}{\beta}} \leq l \leq U \leq 1-Q
$$

which completes the proof of Part (1).

Part (2); Assume that $\{x(n)\}$ is a positive solution of equation (1). We shall prove that $z(n)$ 
does bot have property (i). On the contrary, we assume that $P=\infty$. Then, from (26), we get

$$
\bar{R}_{N}^{\beta}(g(n+\ell)) \omega(n) \geq \bar{R}_{N}^{\beta}(g(n+\ell)) \sum_{s=n}^{\infty} \phi(n+j+s \ell)
$$

Taking limit inferior on both sides as $n \rightarrow \infty$, we get in view of 23 that

$$
1 \geq l \geq P=\infty .
$$

This is a contradiction. Now we admit that $Q=\infty$. Then by (30), $U=-\infty$, which contradicts (23). The proof is now complete.

Theorem 3.6. Assume that (10) and (17) hold. If

$$
P=\liminf _{n \rightarrow \infty} \bar{R}_{n_{0}}^{\beta}(g(n+j+\ell)) \sum_{s=n}^{\infty} \phi(n+j+s \ell)>\frac{\beta^{\beta}}{(\beta+1)^{\beta+1}},
$$

then every solution of Equation (1) is either oscillatory or tends to zero as $n \rightarrow \infty$.

Proof. Let $\{x(n)\}$ be a non-oscillatory solution of equation (11). Without loss of generality we may assume that $x(n)>0$. If $P=\infty$, then by Lemma 3.5, $z(n)$ does not have property (i). That is, $z(n)$ satisfies property (ii). Therefore, from Lemma 3.2 , we have $\lim _{n \rightarrow \infty} x(n)=0$.

Now, Let $P<\infty$. By Lemma 3.1, we have that $z(n)$ has either property (i) or property (ii). If $z(n)$ has the property (ii), from Lemma 2.2, we obtain $\lim _{n \rightarrow \infty} x(n)=0$.

Next, we assume that $z(n)$ holds property (i). Let $\omega$ and $l$ be defined by (15) and (16), respectively. Then from Lemma 3.5 , we have $P \leq l-l^{\frac{\beta+1}{\beta}}$. Using inequality 29 with $u=l$ and $A=B=1$, we get that

$$
P \leq \frac{\beta^{\beta}}{(\beta+1)^{\beta+1}},
$$

which contradicts (31). This completes the proof.

Example 3.7. Consider the third-order neutral delay difference equation

$$
\Delta_{\ell}\left(\frac{1}{n} \Delta_{\ell}\left(\frac{1}{n} \Delta_{\ell}\left(x(n)+\frac{1}{2} x(n-2 \ell)\right)\right)\right)+\frac{3\left(4 n^{3}+10 n^{2} \ell+7 n \ell^{2}+2 \ell^{3}\right)}{n^{2}(n+\ell)^{2}(n+2 \ell)} x(n-2 \ell)=0 .
$$

We note that $\beta=1$ and $f(x)=x$. Hence, it is easy to see that (10) and (17) hold and by Theorem 3.6, we see that every solution of equation (32) is either oscillatory or converges to zero as $n \rightarrow \infty$. In fact, $\{x(n)\}=\left\{(-1)^{\left[\frac{n}{\ell}\right]}\right\}$ is one such solution of equation (32). 
Example 3.8. Consider the third-order neutral delay difference equation

$$
\begin{aligned}
& \Delta_{\ell}\left(n \Delta_{\ell}\left(n(n+\ell) \Delta_{\ell}\left(x(n)+\frac{(n-\ell)^{2}}{n^{2}} x(n-\ell)\right)^{3}\right)\right) \\
& +8 \ell^{7}(n-2 \ell)^{2}\left(\frac{n(2 n+\ell)^{3}}{n^{5}(n+\ell)^{5}}-\frac{(2 n+3 \ell)^{3}(2 n+\ell)}{(n+\ell)^{5}(n+2 \ell)^{5}}+\frac{(n+\ell)(2 n+5 \ell)^{3}}{(n+2 \ell)^{5}(n+3 \ell)^{5}}\right) x(n-2 \ell)=0 .
\end{aligned}
$$

We note that $\beta=3$ and $f(x)=x$. Hence, it is easy to see that (10) and (17) hold and by Theorem 3.6, we see that every solution of equation (42) is either oscillatory or converges to zero as $n \rightarrow \infty$. In fact, $\{x(n)\}=\left\{\frac{\ell^{2}}{n^{2}}\right\}$ is one such solution of equation (42).

Theorem 3.9. Assume that (10) and (17) hold. If

$$
P+Q>1
$$

then every solution of Equation (1) is either oscillatory or tends to zero as $n \rightarrow \infty$.

Proof. Let $x(n)$ be a non-oscillatory solution of equation (1). Let us assume that $x(n)>0$. If $P=\infty$ or $Q=\infty$, then by Lemma 3.5, $z(n)$ does bot have property (i). That is, $z(n)$ satisfies property (ii). Then from Lemma 3.2, we get $\lim _{n \rightarrow \infty} x(n)=0$.

Next, Let $P<\infty$ and $Q<\infty$. By Lemma 3.1, we have $z(n)$ satisfies property (i) or property (ii). If $z(n)$ satisfies property (ii), from Lemma 3.2, we obtain $\lim _{n \rightarrow \infty} x(n)=0$. If for $z(n)$ property (ii) holds, then continuing as above and by lemma 3.2 we obtain $\lim _{n \rightarrow \infty} x(n)=0$. Now, we assume that $z(n)$ satisfies property (i). Then from Lemma 3.5 , we have $P+Q \leq 1$ which contradicts (31) and hence this completes the proof.

Example 3.10. Consider the third-order neutral delay difference equation

$$
\Delta_{\ell}\left(n \Delta_{\ell}\left(\Delta_{\ell}\left(x(n)+\frac{n-\ell}{2 n} x(n-\ell)\right)\right)^{3}\right)+\frac{27 \ell^{7}\left(8 n^{2}+27 n \ell+27 \ell^{2}\right)(n-\ell)^{3}}{n^{2}((n+\ell)(n+2 \ell)(n+3 \ell))^{3}} x^{3}(n-\ell)=0
$$

It is easy to see that all conditions of Theorem 3.9 are satisfied and hence every solution of equation (37) is either oscillatory or converges to zero as $n \rightarrow \infty$.In fact, $\{x(n)\}=\left\{\frac{\ell}{n}\right\}$ is one such solution of equation (37). 
Example 3.11. Consider the third-order neutral delay difference equation

$$
\begin{aligned}
\Delta_{\ell}\left(n ^ { 6 } \Delta _ { \ell } \left(n^{2} \Delta_{\ell}(x(n)\right.\right. & \left.\left.\left.+\frac{1}{n} x(n-\ell)\right)\right)^{3}\right)+\ell^{2}\left(\frac{n^{6}\left(2 \ell^{3}+\ell^{2}(7 n+1)+7 \ell n^{2}+2 n^{3}\right)}{(n-3 \ell)(\ell+n)(2 \ell+n)}\right. \\
& \left.-\frac{(\ell+n)^{7}\left(18 \ell^{3}+\ell^{2}(27 n+1)+13 \ell n^{2}+2 n^{3}\right)}{(n-3 \ell)(n+\ell)(n+2 \ell)(n+3 \ell)}\right) x(n-3 \ell)=0 .
\end{aligned}
$$

We note that $\beta=3$ and $f(x)=x$. It is easy to see that all conditions of Theorem 3.9 are not satisfied and hence every solution of equation (36) is tends to infinity as $n \rightarrow \infty$.

Corollary 3.12. Assume that $(10)$ and $(17)$ hold. If

$$
Q=\limsup _{n \rightarrow \infty} \frac{1}{\bar{R}_{n_{0}}(g(n+j+\ell))} \sum_{s=0}^{\frac{n-n_{0}-j-\ell}{\ell}} \bar{R}_{n_{0}}^{\beta+1}\left(g\left(n_{0}+j+s \ell+\ell\right)\right) \phi\left(n_{0}+j+s \ell\right)>1
$$

then every solution of Equation (1) is either oscillatory or tends to zero as $n \rightarrow \infty$,

Example 3.13. Consider the third-order neutral delay difference equation

$$
\Delta_{\ell}\left(\Delta_{\ell}\left(\Delta_{\ell}\left(x(n)+\frac{1}{3} x(n-\ell)\right)\right)^{3}\right)+q(n) f(x(g(n)))=0, n>\ell .
$$

Here $q(n)=\frac{32 \ell^{7}\left(16 n^{4}+40 n^{3} \ell+13 n^{2} \ell^{2}-30 n \ell+9 \ell^{4}\right)}{3 n^{2}((n+\ell)(n+2 \ell)(n+3 \ell))^{3}}, f(x)=x^{3}$ and $g(n)=n-\ell$. It is easy to see that all conditions of Corollary 3.12 are satisfied and hence every solution of equation (37) is either oscillatory or converges to zero as $n \rightarrow \infty$.In fact, $\{x(n)\}=\left\{\frac{\ell}{n}\right\}$ is one such solution of equation (37).

Theorem 3.14. Let (10) holds. Assume that there exists a positive function $\rho(n)$ such that

$$
\begin{aligned}
\limsup _{n \rightarrow \infty} & \sum_{0}^{\frac{n-n_{0}-j-\ell}{\ell}}\left(\rho\left(n_{0}+j+s \ell\right) \phi\left(n_{0}+j+s \ell\right)\right. \\
& \left.-\frac{\beta^{\beta}}{(\beta+1)^{\beta+1}}\left(\frac{\Delta_{\ell} \rho\left(n_{0}+j+s \ell\right)}{\rho\left(n_{0}+j+s \ell\right)}\right)^{\beta+1} \psi\left(n_{0}+j+s \ell\right)\right)=\infty .
\end{aligned}
$$

where $\psi(n)=\rho(n)\left(\beta R_{n_{0}}(g(n+\ell))\right)^{-\beta}$. Then, every solution of equation (1) is either oscillatory or tends to zero as $n \rightarrow \infty$. 
Proof. Let $x(n)$ be a non-oscillatory solution of equation (1). Without loss of generality we may assume that $x(n)>0$. By Lemma 3.1, we have that $z(n)$ has the proerty (i) or property (ii). If $z(n)$ possess property (ii), from Lemma 3.2, we obtain $\lim _{n \rightarrow \infty} x(n)=0$. Next, let $z(n)$ satisfies the property (i). By Lemma 3.3, we have that (11) and (12) hold. Now, we define

$$
\omega_{1}(n)=\rho(n) \frac{E_{2}(n)}{z^{\beta}(g(n))}
$$

By applying $\Delta_{\ell}$ and using (11) and (12), we have

$$
\Delta_{\ell} \omega_{1}(n) \leq-\rho(n) \phi(n)+\frac{\Delta_{\ell} \rho(n)}{\rho(n+\ell)} \omega_{1}(n+\ell)-\psi^{-\frac{1}{\beta}}(n) w_{1}^{\frac{\beta+1}{\beta}}(n+\ell) .
$$

Using inequality 29 with $u=\omega_{1}(n+\ell), A=\frac{\Delta_{\ell} \rho(n)}{\rho(n+\ell)}$ and $B=\psi^{-\frac{1}{\beta}}(n)$, we obtain

$$
\frac{\Delta_{\ell} \rho(n)}{\rho(n+\ell)} \omega_{1}(n+\ell)-\psi(n)^{-\frac{1}{\beta}} w_{1}^{\frac{\beta+1}{\beta}}(n+\ell) \leq \frac{\beta^{\beta}}{(\beta+1)^{\beta+1}}\left(\frac{\Delta_{\ell} \rho(n)}{\rho(n+\ell)}\right)^{\beta+1} \psi(n)
$$

Therefore, we get

$$
\Delta_{\ell} \omega_{1}(n) \leq-\rho(n) \phi(n)+\frac{\beta^{\beta}}{(\beta+1)^{\beta+1}}\left(\frac{\Delta_{\ell} \rho(n)}{\rho(n+\ell)}\right)^{\beta+1} \psi(n) .
$$

By summing the above inequality from $n_{0}$ to $n-\ell$ we have

$$
\begin{aligned}
\omega_{1}(n) \leq \omega_{1}\left(n_{2}+j\right)-\sum_{s=0}^{n-n_{0}-j-\ell \ell} & \left(\rho\left(n_{0}+j+s \ell\right) \phi\left(n_{0}+j+s \ell\right)\right. \\
& \left.-\frac{\beta^{\beta}}{(\beta+1)^{\beta+1}}\left(\frac{\Delta_{\ell} \rho\left(n_{0}+j+s \ell\right)}{\rho\left(n_{0}+j+s \ell+\ell\right)}\right)^{\beta+1} \psi\left(n_{0}+j+s \ell\right)\right) .
\end{aligned}
$$

Taking limit superior as $n \rightarrow \infty$ and using (38), we have $\omega_{1}(n) \rightarrow-\infty$, which contradicts that $\omega_{1}(n)>0$. This completes the proof of the theorem.

Example 3.15. Consider the third-order neutral delay difference equation

$$
\Delta_{\ell}\left(n \Delta_{\ell}\left(\frac{1}{n} \Delta_{\ell}\left(x(n)+\frac{1}{3} x(n-3 \ell)\right)^{5}\right)\right)+\frac{4 n^{2}+10 n \ell+5 \ell^{2}}{(n+\ell)(n+2 \ell)} x^{5}(n-4 \ell)=0 .
$$

We have $\beta=5$ and $f(x)=x^{5}$. It is easy to see that condition (10) holds. Hence, by Theorem 3.6. we see that every solution of equation (42) is either oscillatory or converges to zero as $n \rightarrow \infty$. In fact, $\{x(n)\}=\left\{(-1)^{\left[\frac{n}{\ell}\right]}\right\}$ is one such solution of equation (32) . 


\section{REFERENCES}

[1] Agarwal. R. P, Difference Equations and Inequalities, Theory, Methods and Applications, 2nd Edition, Marcel Dekker, New York, 2000.

[2] Dosla. D and Kobza. A, Global Asymptotic Properties of Third-order Difference Equations, An International Journal Computers and Mathematics with Applications, 48(2004), 191-200.

[3] Hardy. G.H, Littlewood. J.E and Polya. G, Inequalities, 2nd Edition, Cambridge University Press, 1952.

[4] John R. Graef and Thandapani. E, Oscillatory and Asymptotic Behavior of Solutions of Third Order Delay Difference Equations, Funkcialaj Ekvacioj, 42 (1999), 355-369.

[5] Maria Susai Manuel. M, Britto Antony Xavier. G and Thandapani. E, Theory of generalized difference operator and its applications, Far East Journal of Mathematical science, 20(2)(2006), 163-171.

[6] Maria Susai Manuel. M, Adem Kılıçman, Britto Antony Xavier. G, R. Pugalarasu and Dilip. D. S, On The Solution of Second Order Generalized Difference Equations, Advances in Difference Equations, 105(2012).

[7] Maria Susai Manuel. M, Adem Kılıçman, Britto Antony Xavier. G, R. Pugalarasu and Dilip. D. S, An Application on The Second Order Generalized Difference Equations, Advances in Difference Equations, $35(2013)$.

[8] Pachpatte. B. G, In Equalities for Finite Difference Equations, Marcl Dekker Inc, New York, Basel, 2002.

[9] Popenda. J and Schmeidel. E, Nonoscillatory Solution of Third Order Difference Equations, Portugaliae Mathematica , 49(1992), 233-239.

[10] Ronald E. Mickens, Difference Equations, Van Nostrand Reinhold Company, New York, 1990.

[11] Said R. Grace, Ravi P. Agarwal and John R. Graef, Oscillation Criteria for Certain Third Order Nonlinear Difference Equations, Applicable Analysis and Discrete Mathematics, 3 (1) (2009), 27-38.

[12] Saker. S. H, Alzabut. J. O and Mukheimer. A, On the Oscillatory Behavior for A Certain Class of Third Order Nonlinear Delay Difference Equations, Electronic Journal of Qualitative Theory of Differential Equations, 67(2010), 1-16.

[13] Water G. Kelley and Allan C. Peterson, Difference Equations, An Introduction with Applications, Academic Press, inc 1991. 